Médiation du discours expert : approches

linguistiques

\title{
Isobel Noble, Anglais appliqué. Droit, Science politique
}

Paris : LGDJ Lextenso, 2013

\section{Marion Charret-Del Bove}

\section{(2) OpenEdition}

\section{Journals}

Édition électronique

URL : http://journals.openedition.org/asp/4170

DOI : 10.4000/asp.4170

ISSN : 2108-6354

\section{Éditeur}

Groupe d'étude et de recherche en anglais de spécialité

Édition imprimée

Date de publication : 1 mars 2014

Pagination : 130-133

ISSN : 1246-8185

\section{Référence électronique}

Marion Charret-Del Bove, «Isobel Noble, Anglais appliqué. Droit, Science politique », ASp [En ligne], 65 |

2014, mis en ligne le 01 mars 2014, consulté le 03 novembre 2020. URL : http://

journals.openedition.org/asp/4170; DOI : https://doi.org/10.4000/asp.4170

Ce document a été généré automatiquement le 3 novembre 2020.

Tous droits réservés 


\section{Isobel Noble, Anglais appliqué. Droit, Science politique}

Paris : LGDJ Lextenso, 2013

Marion Charret-Del Bove

\section{RÉFÉRENCE}

Noble, Isobel. 2013. Anglais appliqué. Droit, Science politique. Paris : LGDJ Lextenso. 292 p.

ISBN: 978-2-275-04119-3. 
1 Anglais appliqué est la troisième édition d'un manuel destiné principalement aux étudiants de Licence et Master qui suivent un enseignement d'anglais de spécialité dans les disciplines de Droit et Science politique, ainsi qu'aux étudiants des BiLicences, des IEP, des écoles de commerce et de journalisme. Dans l'introduction, l'auteure rappelle que l'objectif de cet ouvrage est, par le biais d'une approche active, de "fournir un outil de travail structurant l'apprentissage de la langue de spécialité » (p. xii). Il s'agit de permettre au public visé d'acquérir des connaissances dans les domaines juridique, politique et philosophique tout en s'appropriant des compétences langagières orales et écrites en langue de

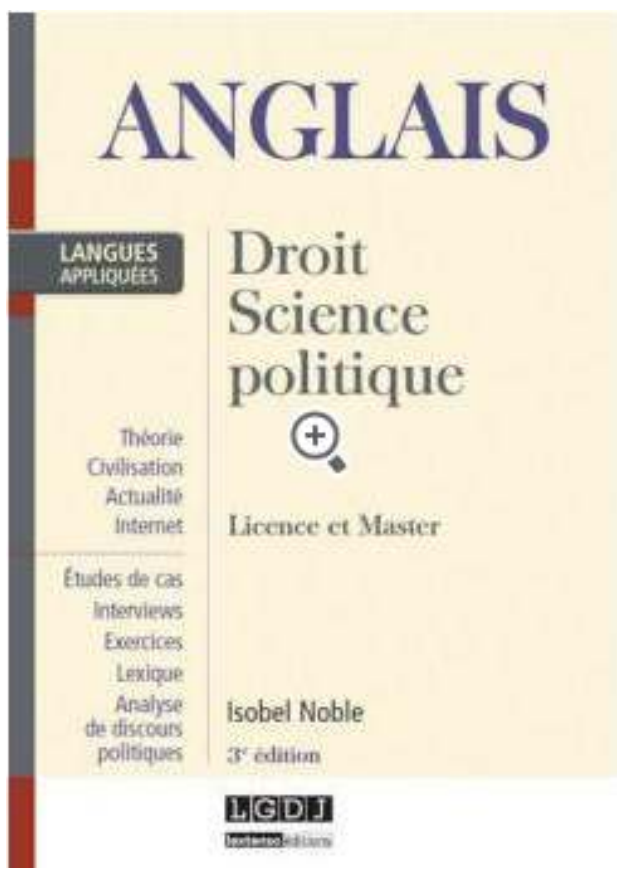
spécialité.

2 L'ouvrage, entièrement rédigé en anglais à l'exception de l'introduction, est divisé en sept parties intitulées respectivement "Common law systems", "Law-making and law enforcement ", "Freedoms, rights and duties ", "Political systems in the UK", "Political philosophy ", " Political Discourse: political speech in action » et "Interviews: Law in action ». De ce fait, les thématiques suivantes sont abordées: les origines et les principes fondamentaux de la common law, l'élaboration et l'application de la loi, les droits de l'homme et de la personne, le système politique au Royaume-Uni, la philosophie et la rhétorique politiques, le système juridique anglais actuel décrit par des juristes. Les cinq premières parties regroupent vingt et un chapitres. La partie 6 se compose de quatre sous-parties, chacune intitulée «Speech", tandis que la dernière partie comporte cinq sous-parties nommées « Interview ». L'ouvrage se termine par deux index (lexical et grammatical) dont les références renvoient aux numéros de chapitres.

Les parties 1 à 5 sont structurées de manière identique. En premier lieu, l'auteure explique brièvement dans deux rubriques «In this part you will read about " et "You will learn some basic legal terms concerning" les thématiques abordées ainsi que les connaissances du domaine de spécialité qui vont être acquises par la suite. Dans un second temps, la première page de chaque chapitre fournit une liste de mots, "Key words and concepts ", que le lecteur va rencontrer de manière linéaire dans le texte de base mis en valeur par un cadre gris. Les mots et expressions en gras de cette liste font ensuite l'objet de définitions précises et claires dans la rubrique «Definitions ». Puis, I. Noble propose des exercices de compréhension, de vocabulaire, de grammaire et d'expression écrite se référant au premier texte. Figurent également un ou deux autres documents de support accompagnés d'exercices de compréhension, de manipulation lexicale ainsi que d'activités de discussion.

Dans les parties 6 et 7, l'auteure liste tout d'abord les compétences langagières visées et la méthodologie suivie pour les acquérir. La partie 6 est consacrée à l'analyse de discours politiques et de la rhétorique qui les sous-tend. Le lecteur est invité à remplir une grille de compréhension type, puis à mobiliser les compétences acquises dans le 
cadre de débats collectifs et d'exercices de production écrite. De manière originale, I. Noble propose dans la partie 7 de travailler à partir d'entretiens conduits par ellemême auprès d'acteurs du monde judiciaire de common law afin de mieux saisir les particularités de ce système.

5 L'auteure de ces lignes a détecté quelques coquilles; par exemple, il manque un it à la page 75 . La police de caractères de l'exercice 1 (A) n'est pas la même à la page 131. La source du texte à la page 177 n'est pas intégrée dans le cadre gris et le titre de l'ouvrage n'est pas souligné à la page 183. Néanmoins, ces coquilles sont mineures et l'ouvrage présente de nombreux atouts dont le premier réside dans la très grande variété des textes de support proposés. En effet, I. Noble a soigneusement sélectionné des extraits de genres différents: des ouvrages de doctrine en droit anglais et américain, des articles de presse, des textes de loi et des extraits de la constitution américaine, des discours émanant de membres de la sphère judiciaire, des fiches d'arrêt ainsi que des passages d'œuvres littéraires telles que les Selected Short Stories de Graham Greene ou The Adventures of Robin Hood de R. Lancey Green. Dans la partie 5, les textes de support sont tirés d'ouvrages philosophiques écrits par Thomas Paine, Thomas Hobbes et J.S. Mill ; ils pourront constituer du matériel pédagogique innovant pour l'enseignant de langue de spécialité dans la filière Droit-Philosophie. La partie 7 intègre les transcriptions d'entretiens menés auprès de membres des professions juridiques en Angleterre parmi lesquels figurent, entre autres, un magistrate et un district judge, un solicitor et un barrister. Ces textes apportent indéniablement non seulement un éclairage sur l'évolution actuelle du monde juridique de common law, mais aussi des informations supplémentaires d'ordre culturel par exemple à propos du rôle joué dans la société anglaise par le juge.

6 La diversité des exercices proposés constitue le second point fort de ce livre. Afin d'évaluer la compréhension des textes, l'auteure propose des activités de reformulation, de remise en ordre chronologique, des exercices "vrai-faux» et des QCM. Il faut aussi parfois relier des dates et des événements, ou des amorces de phrases avec la fin correspondante. Pour favoriser l'apprentissage du lexique, le lecteur doit retrouver, dans le texte de base, des synonymes ou des antonymes d'expressions données, compléter des phrases ou un texte grâce aux mots définis dans le glossaire ou encore reconstituer des collocations, par exemple to withhold evidence, to take an oath, to plead guilty, etc. Différents points grammaticaux, énumérés dans le second index, sont abordés par le biais d'exercices toujours contextualisés. Le lecteur peut ainsi travailler sur la voix passive, les modaux, les temps du passé en particulier avec for, since, ago, during, les phrases interrogatives, les indénombrables, les prépositions et les verbes à particules par le biais principalement d'exercices de remplissage et de reformulation.

7 À tous ces exercices s'ajoutent de nombreuses activités de rédaction. Il s'agit pour le lecteur de comparer, de finir une histoire ou d'écrire un dialogue, de rédiger un entretien, un article de presse ou le portrait de quelqu'un en évitant soigneusement les répétitions. Le lecteur peut également être amené à exprimer, de manière structurée, une opinion motivée relative aux questions abordées. À cet effet, la fiche d'expressions mobilisables dans le cadre d'essais, de commentaires ou de résumés de la page 68 peut s'avérer utile. En dernier lieu, l'auteure inclut dans l'ouvrage des activités de recherches sur internet invitant le lecteur à consulter des sites pour en savoir plus sur la législation antiterroriste au Royaume-Uni ou sur la discrimination homme-femme (arrêt Reed v. Reed p. 65) et participer à des discussions sur ces sujets d'actualité. Des 
exercices d'expression orale (individuelle ou collective) sont suggérés tout au long de l'ouvrage portant sur des thématiques variées allant du rôle du jury populaire au mariage homosexuel en passant par la liberté d'expression, la réforme de la Chambre des Lords, les politiques pénales (durée de la détention, tolérance zéro) et les dangers d'internet.

8 Le troisième élément novateur de ce livre se trouve dans les activités d'analyse stylistique régulièrement proposées au lecteur qui doit travailler sur les stratégies argumentatives, stylistiques et rhétoriques présentes dans les différents textes de support. À la fin de la partie 6 consacrée aux discours politiques, figure une grille de lecture dans laquelle une série de questions permet au lecteur de repérer les éléments suivants : locuteur, contexte, ton, public visé, buts recherchés, moyens langagiers, thèmes traités, références culturelles, place du raisonnement et des émotions dans le discours. Cette approche déductive vise dans un premier temps à repérer les principes de raisonnement et de rhétorique au cœur des textes étudiés pour, dans un second temps, s'approprier et réutiliser ces stratégies d'expression écrite et orale.

9 Cependant, certaines critiques peuvent être formulées à l'égard de cet ouvrage. Tout d'abord, on peut regretter la présence d'un index se référant uniquement aux numéros de chapitres. Même si l'auteure indique que ce choix repose sur une volonté de faciliter une vérification rapide des mots employés, l'inclusion des références aux numéros de page aurait permis de renforcer l'apprentissage lexical en contexte. De même, I. Noble a choisi de ne pas spécifier la nature grammaticale des mots donnés sur la première page de chaque chapitre, ce qui peut rendre l'appropriation et la réutilisation de ces mots plus laborieuses. La présentation adoptée pour cette première liste de mots suppose que le lecteur opère un constant va-et-vient entre ladite liste et le texte au verso de la même page, texte dont la lecture peut en être ralentie. Il est également regrettable que, pour certains textes de support entièrement rédigés par l'auteure comme aux pages 12 et 13, celle-ci n'ait pas mentionné les sources consultées. Enfin, dans les références aux décisions de justice émanant de la common law anglaise contrairement au système américain, le $v$ (abréviation du latin versus) n'est pas suivi d'un point; il s'agit de la norme de présentation recommandée par le Oxford Standard for Citation of Legal Authorities (OSCOLA 2006).

De plus, il est clair à l'analyse des différents documents que l'utilisation d'un tel manuel présuppose une bonne maîtrise de la langue anglaise générale que trop peu d'étudiants de première et deuxième années de licence possèdent. En raison de la présence assez rare de conseils pratiques et en l'absence de corrigés des divers exercices ou activités proposés, cet ouvrage ne pourra véritablement être utilisé en totale autonomie que par une partie restreinte du public ciblé. En revanche, l'auteure précise après son introduction que « les collègues qui le désirent peuvent demander un corrigé auprès de l'auteur en justifiant de leur qualité d'enseignant» (p.xii). De ce fait, ce manuel constitue pour le professeur d'anglais un véritable outil pédagogique dans lequel il pourra sélectionner une multitude d'activités afin d'élaborer ses séquences d'enseignement pour des non-spécialistes. En outre, certains exercices nécessitent assurément la présence de l'enseignant pour vérifier la construction syntaxique d'expressions telles que whereas et unlike aux pages 10 et 68 . Parfois, plusieurs points grammaticaux sont abordés au sein de la même activité comme à la page 153 où il s'agit d'indiquer le temps approprié tout en formulant des phrases passives et négatives, ce qui suppose la maîtrise grammaticale de trois difficultés en même temps. Si, en début 
d'ouvrage, les exemples sont assez rares et rendent la réalisation d'exercices plus difficile comme ceux sur les modaux aux pages 20 et 21 , les phrases d'amorces sont plus nombreuses aux pages 142 et 170 , prouvant que l'auteure a pleinement conscience des possibles difficultés rencontrées par le lecteur.

11 Ces remarques ponctuelles n'atténuent pas cependant les éloges qu'il faut adresser à I. Noble pour avoir su élaborer un ouvrage si clair et si riche. Le format choisi se prête aussi bien à la consultation par thème qu'à la lecture complète. L'apport linguistique et culturel de l'Anglais appliqué est immense pour ceux qui l'utiliseront partiellement ou totalement.

\section{BIBLIOGRAPHIE}

Oxford Standard for Citation of Legal Authorities (OSCOLA). 2006. <www.law.ox.ac.uk/published/ oscola/oscola_2006.pdf>. Consulté le 21 janvier 2014.

\section{AUTEURS}

\section{MARION CHARRET-DEL BOVE}

Faculté de droit et de sciences politiques, Université Jean Moulin Lyon 3. marion.del-bove@univlyon3.fr 